\title{
Technical nuances of clipping a middle cerebral artery bifurcation aneurysm
}

\author{
Piyush Kalakoti, M.D., Shyamal C. Bir, M.D., Ph.D., Richard D. Murray, M.D., Osama Ahmed, M.D., \\ and Anil Nanda, M.D., MPH
}

Department of Neurosurgery, Louisiana State University, Shreveport, Louisiana

Broad-necked middle cerebral artery aneurysms present unique challenges for the vascular neurosurgeon, who must contend with smaller vessels and often a complex clipping strategy. Due to their superficial location, these lesions are still commonly selected for microsurgical clipping. We present a case of a 42-year-old woman with significant vascular disease with a right middle cerebral artery aneurysm. We discuss the key surgical steps, demonstrate the microsurgical dissection and intraoperative rupture encountered and the final clipping strategy, as well as the postoperative course in this operative video presentation.

The video can be found here: http://youtu.be/qZ2gvqz7XdQ.

KEYWORDS MCA bifurcation; aneurysm; clipping; video 